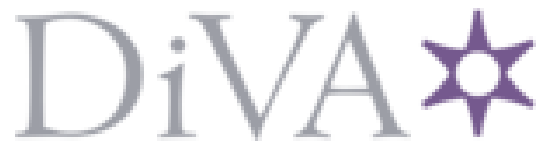

http://www.diva-portal.org

Preprint

This is the submitted version of a paper presented at 2019 57th Annual Allerton Conference on Communication, Control, and Computing (Allerton), 24-27 Sept. 2019.

Citation for the original published paper:

Zhou, L., Vu, M T., Oechtering, T J., Skoglund, M. (2019)

Fundamental Limits for Biometric Identification Systems without Privacy Leakage In: Proceedings 57th Annual Allerton Conference on Communication, Control, and Computing (Allerton) IEEE

https://doi.org/10.1109/ALLERTON.2019.8919895

N.B. When citing this work, cite the original published paper.

The work was supported by the Swedish Research Council (VR) project under Grant 2016-03853.

Permanent link to this version:

http://urn.kb.se/resolve?urn=urn:nbn:se:kth:diva-271127 


\title{
Fundamental Limits for Biometric Identification Systems without Privacy Leakage
}

\author{
Linghui Zhou, Minh Thanh Vu, Tobias J. Oechtering, and Mikael Skoglund \\ Division of Information Science and Engineering \\ KTH Royal Institute of Technology
}

\begin{abstract}
We study the biometric identification systems without privacy leakage. Privacy-preserving biometric identification systems that involve helper data, secret keys and private keys are considered. The helper data are stored in a public database and can be used to support the user identification. The secret key is either stored in an encrypted database or handed to the user, which can be used for authentication. Since the helper data are public and releasing the biometric information invokes privacy issues, the public helper data can only leak a negligible amount of biometric information. To achieve this, we use private keys to mask the helper data such that the public helper data contain as little as possible information about the biometrics. Moreover, a two-stage extension is also studied, where the clustering method is used such that the search complexity in the identification phase can be reduced. identification
\end{abstract}

\section{INTRODUCTION}

Biometric identification systems are more and more used in various applications as they can ensure a higher level of security and convenience compared to traditional identification systems, e.g. keys, tokens, cards, etc. The physical features that the biometric identification systems are using, such as face, fingerprint and iris, are unique for each individual and do not significantly change over time. However, the uniqueness of biometrics also invoke privacy issue. As pointed out in [1], once biometric information is stolen and compromised, there is no way to get it back. Therefore, privacy is a significant aspect that has to be taken into account in the design of biometric identification systems.

Willems et al. characterized in [2] the capacity of a biometric identification system. Tuncel et al. analyzed in [3] the capacity and the storage tradeoff in a multi-stage biometric identification system, where the biometric data are firstly compressed and then enrolled in the database. In [4], multistage stage identification systems are studied to reduce search complexity. Moreover, hierarchical identification considering pre-processing is further discussed in [5], which provides another way of an efficient system architecture. Further, in [6], several assumptions in the identification problem are relaxed and the corresponding fundamental bounds regions are derived.

The compression operation ensures privacy protection to some extent as the stored data are noisy. However, this is not effective enough because it was found that the distorted data still contains biometric information [7]. The privacy and secrecy aspects of biometric systems are investigated in [8].

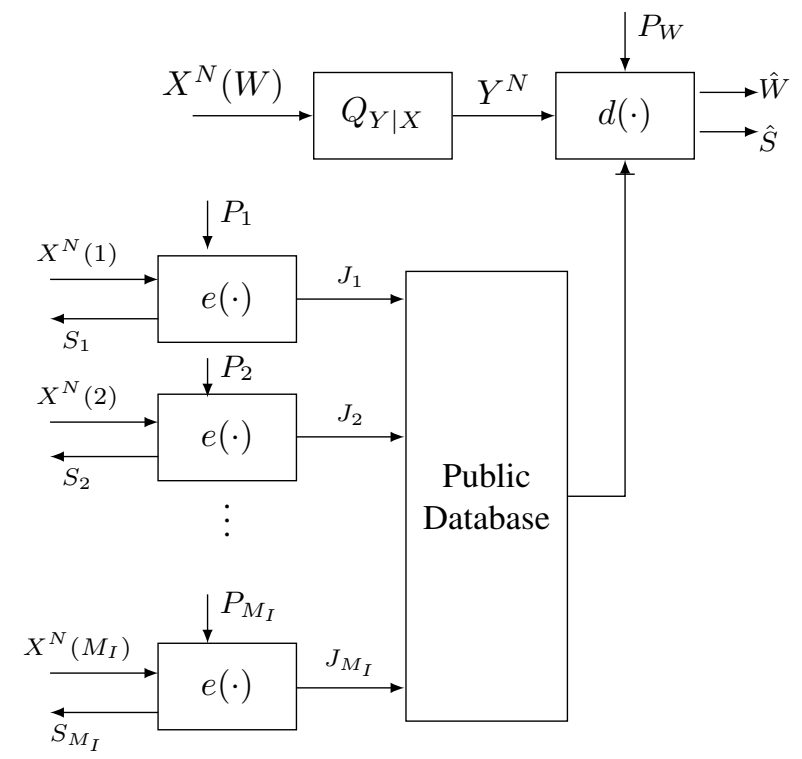

Fig. 1. An overview of a biometric identification system without privacy leakage. Assume a previously enrolled user $W$ is observed via a memoryless channel $Q_{Y \mid X}$ which produces the observation $Y^{N} . W$ is uniformly distributed over $\left[1: M_{I}\right]$ and independent of users' data. The public database stores the helper data $\boldsymbol{J}=\left(J_{i}\right)_{i=1}^{M_{I}}$.

Fundamental limits of biometric identification systems that preserve privacy and enable authentication are studied in [9], where the privacy is preserved in the sense that the public helper data leak no information about the secret key, while it still leak information about the biometrics.

The motivation of this work is to explore the biometric identification systems such that the public helper data only leak negligible information about the secret key and biometrics, respectively. Our approach resembles the one in [8]. To achieve this, we use private keys to mask the helper data as in [8]. We next extend the consideration to a two-stage setting using the clustering method which leads to a reduction of the identification search complexity.

The proposed biometric identification system consists of two phases: the enrollment phase and the identification phase. The enrollment phase uses users' data and the private keys to generate helper data and secret keys. The private keys provided by some trusted source are used to mask the helper data and are handed to each user, e.g. stored in an access card or token. The helper data are stored in a 


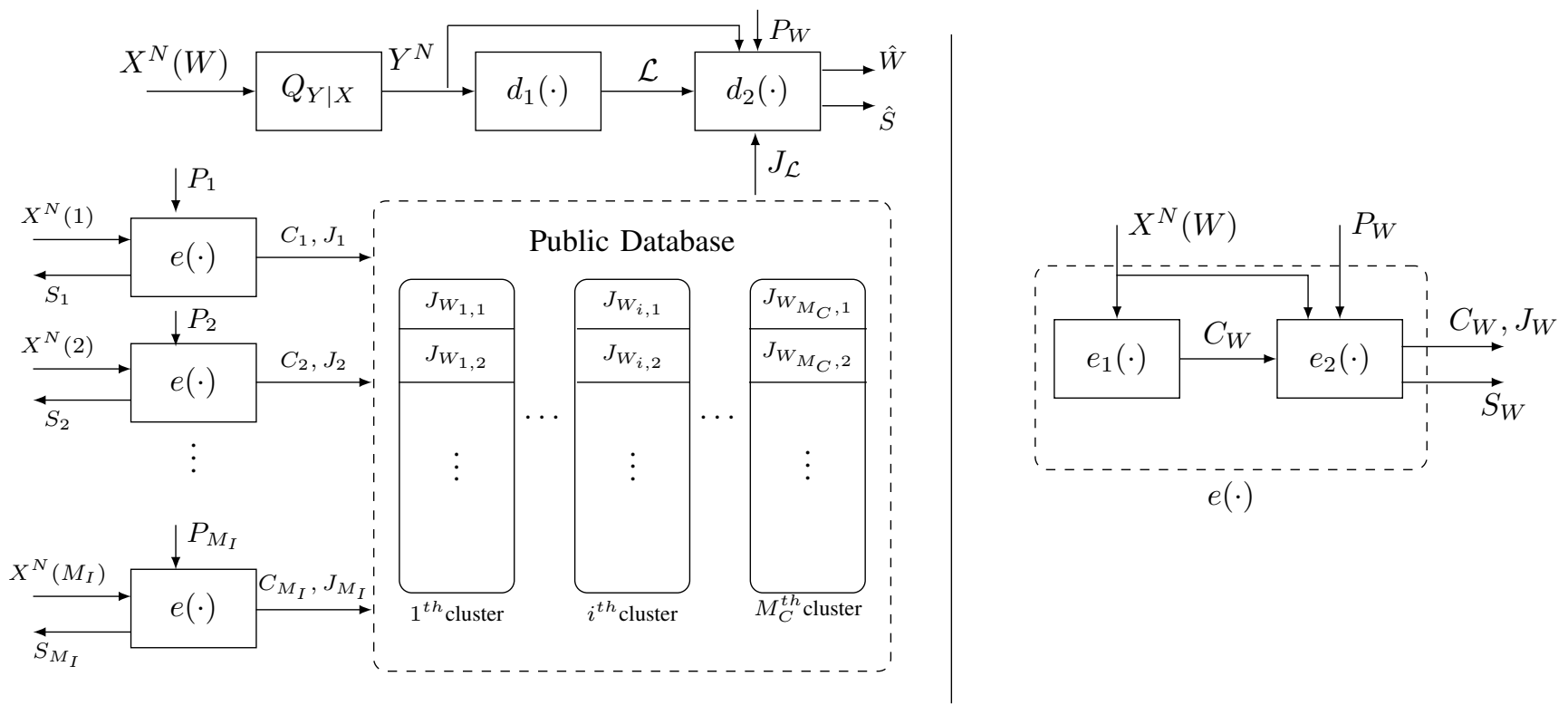

Fig. 2. Model for a two-stage biometric identification system without privacy leakage. The enrollment and identification phases are both finished in two steps. In the enrollment phase, the encoder $e_{1}(\cdot)$ uses the biometric sequence $x^{N}(W)$ to decide which cluster the user belongs to and outputs the cluster index $C_{W}$. Then the encoder $e_{2}(\cdot)$ uses the cluster index, biometric data and the private key to generate the secret key $S_{W}$ and the helper data $J_{W}$. In this figure, we use $W_{i, j}$ to denote the $j$-th user in the $i$-th cluster. In the identification phase, the decoder $d_{1}(\cdot)$ uses the observation $Y^{N}$ to estimate a list of cluster indices. Then the decoder $d_{2}(\cdot)$ estimates the user identity and the secret key use the observation $Y^{N}$, the private key $P_{W}$ and the helper data in the list.

public database, available also to the adversaries, and used to support the user identification. The secret key is either stored in an encrypted database or handed to each user, and is used for authentication, i.e., regulate access to sensitive data or services. In the identification phase, a random user presents its (noisy) data and the corresponding private key to the processing unit. The system needs to return the true identity of the user as well as the secret key with high probability. The architecture of the proposed method is similar to that in [9], while the difference is that we include private keys and the privacy constraint is more strict.

The rest of the paper is organized as follows. In Section II, we describe the problem formulation. In Section III, the main results are presented.

Notations: Random variables, their realizations and alphabets are denoted by upper cases, lower cases and calligraphic letters. Vector $\left(X_{1}, X_{2}, \ldots, X_{N}\right)$ is denoted by $X^{N} . H(X)$, $I(X ; Y), Q_{X}(x)$ and $Q_{Y \mid X}(y \mid x)$ represent the entropy, mutual information, marginal and conditional probability distributions respectively. The strong typical set is denoted by $\mathcal{T}_{\epsilon}^{N}$. For a set $\mathcal{A}$, we use $|\mathcal{A}|$ to denote its cardinality.

\section{Problem Formulation}

In this section, we describe the framework of the biometric identification systems for both the single-stage and two-stage settings.

\section{A. Single-Stage Setting}

A model of an identification system is depicted in Fig. 1. Assume that there are $M_{I}$ users indexed by $w \in[1$ : $\left.M_{I}\right]$. The biometric sequence $x^{N}(w)$ of user $w$ is identically independently distributed (i.i.d.) according to $Q_{X}(\cdot)$ on a finite alphabet $\mathcal{X}$, i.e.,

$$
\operatorname{Pr}\left\{X^{N}(w)=x^{N}\right\}=\prod_{i=1}^{N} Q_{X}\left(x_{i}\right),
$$

for all $x^{N} \in \mathcal{X}^{N}$ and $w \in\left[1: M_{I}\right]$.

In the enrollment phase, for each user $w$, the encoder $e(\cdot)$ encodes the biometric sequence $x^{N}(w)$ and the private key $p_{w}$ to generate a helper data index $j_{w}$ and a secret key $s_{w}$, i.e.,

$$
\left(j_{w}, s_{w}\right)=e\left(x^{N}(w), p_{w}\right), .
$$

where $j_{w} \in\left[1: M_{J}\right]$ and $s_{w} \in\left[1: M_{S}\right]$. The private key for each user is independent and uniformly distributed on $\left[1: M_{P}\right]$, hence

$$
\operatorname{Pr}\left\{P_{w}=p\right\}=\frac{1}{M_{P}}, \text { for all } w \in\left[1: M_{I}\right] .
$$

Moreover, this private key is also accessible to the identification phase when the user is trying to access the system.

In the identification phase an unknown user $W$ is observed via a discrete memoryless channel (DMC) $Q_{Y \mid X}(\cdot)$, where $W$ is uniformly distributed over $\left[1: M_{I}\right]$. Hence

$$
\operatorname{Pr}\{W=w\}=\frac{1}{M_{I}} \text {, for all } w \in\left[1: M_{I}\right] .
$$

If user $w$ was observed, the sequence $y^{N}$ occurs with probability

$$
\operatorname{Pr}\left\{Y^{N}=y^{N} \mid X^{N}(w)=x^{N}\right\}=\prod_{i=1}^{N} Q_{Y \mid X}\left(y_{i} \mid x_{i}\right) .
$$


Lastly, after an unknown user with index $w$ is observed, the observation $y^{N}$ and its private key $p_{w}$ are presented to the decoder. The decoder $d(\cdot)$ tries to identify the user index denoted by $\hat{w}$ and the secret key denoted by $\hat{s}$, i.e.,

$$
(\hat{w}, \hat{s})=d\left(y^{N}, \boldsymbol{j}, p_{w}\right)
$$

where $\boldsymbol{j}=\left(j_{i}\right)_{i=1}^{M_{I}}$.

\section{B. Two-Stage Setting}

Next we introduce a two-stage setting, in which the enrollment and the identification processes are divided in two steps. The setting is illustrated in Fig. 2. In the enrollment phase, the encoder first identifies a cluster index for each user

$$
c_{w}=e_{1}\left(x^{N}(w)\right),
$$

and put the user index $w$ inside $\mathcal{B}\left(c_{w}\right)$, where $\mathcal{B}\left(c_{w}\right)$ a set of user indices with cluster index $c_{w}$. Then the encoder generates a helper data sequence and a secret key, using the biometric sequence, the cluster index and the user's private key, i.e.,

$$
\left(j_{w}, s_{w}\right)=e_{2}\left(x^{N}(w), c_{w}, p_{w}\right)
$$

where $j_{w} \in\left[1: M_{J}\right]$ and $s_{w} \in\left[1: M_{S}\right]$. The helper data index $j_{w}$ is stored in the $c_{w}$-th cluster at the location of $w$. Similar to the single-stage setting, the private key $p_{w}$ is also accessible to the decoding operations when the user $w$ is trying to access the system.

In the identification phase, the decoder first decides on a list of cluster indices

$$
\mathcal{L}=d_{1}\left(y^{N}\right)
$$

then the decoder estimates the user index and the secret key

$$
(\hat{w}, \hat{s})=d_{2}\left(y^{N},\left(\boldsymbol{j}_{\mathcal{L}}, \mathcal{B}_{\mathcal{L}}, \mathcal{L}\right), p_{w}\right),
$$

where $\left(\boldsymbol{j}_{\mathcal{L}}, \mathcal{B}_{\mathcal{L}}, \mathcal{L}\right)=\left\{\left(j_{w}, w, c\right) \mid w \in \mathcal{B}(c), c \in \mathcal{L}\right\}$.

\section{Definition}

We define the achievability of a rate tuple for both singlestage and two-stage settings as follows.

Definition 1: An identification, secret key, and private key rate tuple $\left(R_{I}, R_{S}, R_{P}\right) \in \mathbb{R}_{+}^{3}$ is achievable if for all $\delta>0$ there exists enrollment and identification mappings such that for large enough $N$ the following conditions are satisfied

$$
\begin{gathered}
\operatorname{Pr}\left\{(\hat{W}, \hat{S}) \neq\left(W, S_{W}\right)\right\} \leq \delta, \\
\log \left(M_{I}\right) \geq N\left(R_{I}-\delta\right), \\
H\left(S_{W}\right)+N \delta \geq \log \left(M_{S}\right) \geq N\left(R_{S}-\delta\right), \\
\log \left(M_{P}\right) \leq N\left(R_{P}+\delta\right), \\
I\left(S_{W} ; J_{W}\right)+I\left(X^{N}(W) ; J_{W}\right) \leq N \delta .
\end{gathered}
$$

Moreover, we use $\mathcal{R}^{S}$ and $\mathcal{R}^{T}$ to denote the region of all achievable $\left(R_{I}, R_{S}, R_{P}\right)$ for the single-stage and the twostage setting respectively.

The defined capacity region can be interpreted as follows: (11a) indicates that the identification system should be able to return the true user identity and the secret key with only negligible error probability; (11b), (11c) and (11d) define the identification, secret and private key rate respectively; (11e) ensures the identification system is secrecy and privacypreserving in the sense that the helper data leak only negligible information about the secret key and the biometrics.

\section{StATEMENT OF RESUlTS AND PROOFS}

\section{A. Main Results}

In this section, we present the main results of both the single-stage and the two-stage settings, and the proofs in Sections III-B and III-C.

Theorem 1: For a single-stage identification system without privacy leakage, the achievable region is given by

$$
\begin{aligned}
\mathcal{R}^{S}=\{ & \left(R_{I}, R_{S}, R_{P}\right): \\
& R_{I}+R_{S} \leq R_{P}+I(U ; Y) \\
& R_{I}+I(U ; X) \leq R_{P}+I(U ; Y), \\
& \text { for some } P_{U X Y}=Q_{X} Q_{Y \mid X} P_{U \mid X} \\
& \text { and }|\mathcal{U}| \leq|\mathcal{X}|+1\}
\end{aligned}
$$

Theorem 2: For a two-stage identification system without privacy leakage, the achievable region is given by

$$
\begin{aligned}
\mathcal{R}^{T}=\{ & \left(R_{I}, R_{S}, R_{P}\right): \\
& R_{I}+R_{S} \leq R_{P}+I(U, V ; Y), \\
& R_{I}+I(U, V ; X) \leq R_{P}+I(U, V ; Y), \\
& \text { for some } P_{U V X Y}=Q_{X} Q_{Y \mid X} P_{U V \mid X}, \\
& \text { and }|\mathcal{U}| \leq|\mathcal{X}|+1,|\mathcal{V}| \leq|\mathcal{U}||\mathcal{X}|+1\}
\end{aligned}
$$

Based on Theorem 1 and Theorem 2, we have the following results. Fix $V$ to constant in $\mathcal{R}^{T}$, then $\mathcal{R}^{T}=\mathcal{R}^{S}$. This verifies that the single-stage setting is a special case of the two-stage setting. If we restrict ourselves to $R_{I}=0$, then the achievable region is reduced to the region of biometric authentication without privacy leakage derived in [8]. Therefore, authentication is a special case of identification with one enrolled user in the identification system which supports authentication. Moreover, if $\left(R_{I}, R_{S}, R_{P}\right) \in \mathcal{R}^{S}$, then for any $r_{1} \geq 0, r_{2} \geq 0,\left(R_{I}+r_{1}, R_{S}+r_{2}, R_{P}+r_{1}+r_{2}\right) \in \mathcal{R}^{S}$ holds. This can be interpreted as a rate transfer argument. Any extra private key can be used as secret key or to increase the identification rate. This also shows that there is a tradeoff between the identification and secret key rate.

\section{B. Proof of Theorem 1}

1) Achievability: We fix a conditional probability distribution $P_{U \mid X}$. Thus there is $P_{U X Y}=Q_{X} Q_{Y \mid X} P_{U \mid X}$. Let the number of enrolled users be $M_{I}=2^{N R_{I}}$. Additionally, fix a $\delta>0$.

Codebook generation: Pick a rate triple $\left(R_{I}, R_{S}, R_{P}\right) \in \mathcal{R}^{S}$ such that $R_{P} \geq R_{I}+I(U ; X)-$ $I(U ; Y)+3 \delta$ and $R_{S}=R_{P}-R_{I}+I(U ; Y)-2 \delta$. Generate $2^{N R_{c}}$ i.i.d. codewords $u^{N}(k)$ according to $P_{U}$, where we fix $R_{c}=I(U ; X)+\delta$. For each $k \in\left[1: 2^{N R_{c}}\right]$, generate a helper data label $j(k)$ and secret label $s(k)$, where $j(k)$ is generated from $\left[1: 2^{N R_{J}}\right]$ uniformly at random and $s(k)=k$. Further, we fix $R_{J}=R_{I}+I(U ; X)-I(U ; Y)+3 \delta$. 
Enrollment: For each user $w \in\left[1: 2^{N R_{I}}\right]$, the encoder looks for a codeword $u^{N}\left(k_{w}\right)$ such that $\left(u^{N}\left(k_{w}\right), x^{N}(w)\right) \in$ $\mathcal{T}_{\epsilon}^{N}$. If no such $k_{w}$ exists, randomly choose a codeword index from $\left[1: 2^{N R_{c}}\right]$. If there is more than one such $k_{w}$, randomly choose one from them. Moreover, given the private key of this user $p_{w}$, we divide it into two parts, $\bar{p}_{w}$ and $\tilde{p}_{w}$, such that $p_{w}=\left(\bar{p}_{w}, \tilde{p}_{w}\right), \bar{p}_{w} \in\left[1: 2^{N R_{J}}\right]$ and $\tilde{p}_{w} \in\left[1: 2^{N\left(R_{P}-R_{J}\right)}\right]$. Then the encoder produces the corresponding helper data label $j\left(k_{w}\right)$, which is masked by $\bar{p}_{w}$ so that we obtain $j_{w}=$ $j\left(k_{w}\right) \oplus \bar{p}_{w}$. The helper data index $j_{w}$ is stored in the database at the location $w$. A secret key $s_{w}$ is also generated. Let the secret key be generated as $s_{w}=\left(s(k), \tilde{p}_{w}\right)$.

Identification: Upon receiving the observation $y^{N}$ and having access to the private key $p=(\bar{p}, \tilde{p})$ of the observed user $w$, the decoder looks for a unique pair $(\hat{w}, \hat{k})$ such that $j_{\hat{w}}=j(\hat{k}) \oplus \bar{p}$ and $\left(u^{N}(\hat{k}), y^{N}\right) \in \mathcal{T}_{\epsilon}^{N}$. An estimate of the secret key is given by $\hat{s}=(s(\hat{k}), \tilde{p})$.

Error Events Analysis: Assume that user $W$ is observed. Let $K_{W}$ and $J\left(K_{W}\right)$ be the index of the chosen codeword and the helper data label determined by the encoder. Let $P_{W}, S_{W}$ and $J_{W}$ be the actual private key, secret key and helper data stored in the database. Now we define the following error events:

$$
\begin{aligned}
\mathcal{E}_{1}= & \left\{\left(U^{N}(k), X^{N}(W)\right) \notin \mathcal{T}_{\epsilon}^{N}, \forall k \in\left[1: 2^{N R_{c}}\right]\right\}, \\
\mathcal{E}_{2}=\{ & \left.\left(U^{N}\left(K_{W}\right), Y^{N}\right) \notin \mathcal{T}_{\epsilon}^{N}\right\}, \\
\mathcal{E}_{3}=\left\{\exists \hat{w} \neq W,\left(U^{N}\left(K_{W}\right), Y^{N}\right) \in \mathcal{T}_{\epsilon}^{N},\right. & \left.J_{\hat{w}}=J\left(K_{W}\right) \oplus \bar{P}_{W}\right\}, \\
\mathcal{E}_{4}=\left\{\exists(\hat{k}, \hat{w}), \hat{k} \neq K_{W}, \hat{w} \neq W,\right. & \left.\left(U^{N}(\hat{k}), Y^{N}\right) \in \mathcal{T}_{\epsilon}^{N}, J_{\hat{w}}=J(\hat{k}) \oplus \bar{P}_{W}\right\} .
\end{aligned}
$$

If none of the events occur, the identification will be successful, thus we have the following identification error event

$$
\mathcal{E}=\mathcal{E}_{1} \cup \mathcal{E}_{2} \cup \mathcal{E}_{3} \cup \mathcal{E}_{4} .
$$

Since $R_{c}>I(U ; X)$, we obtain that $\operatorname{Pr}\left(\mathcal{E}_{1}\right) \rightarrow 0$ as $N \rightarrow \infty$ due to the covering lemma [10, Lemma 3.3]. By the Markov lemma [10, p.27], we have $\operatorname{Pr}\left(\mathcal{E}_{1}^{c} \cap \mathcal{E}_{2}\right) \rightarrow 0$ as $N \rightarrow \infty$. In the following error events analysis, due to the symmetry and without losing generality, we consider the case when the user index is fixed to $W=1$. The probability $\operatorname{Pr}\left(\mathcal{E}_{3} \mid W=1\right)$ can be bounded as

$$
\begin{aligned}
\operatorname{Pr}\left(\mathcal{E}_{3} \mid W=1\right) \leq & \sum_{\hat{w} \neq 1} \sum_{\bar{p}} \sum_{j} \operatorname{Pr}\left\{J_{\hat{w}}=\bar{p} \oplus j\right\} \\
& \quad \times \operatorname{Pr}\left\{\bar{P}_{1}=\bar{p}, J\left(K_{1}\right)=j\right\} \\
\leq & 2^{N\left(R_{I}-R_{J}\right)}
\end{aligned}
$$

Since $R_{J}>R_{I}$, we obtain that $\operatorname{Pr}\left(\mathcal{E}_{3} \mid W=1\right) \rightarrow 0$ as $N \rightarrow \infty$.

Consider $\operatorname{Pr}\left(\mathcal{E}_{4} \mid W=1\right)$ when $N$ is sufficiently large enough, we can obtain that

$$
\begin{aligned}
& \operatorname{Pr}\left(\mathcal{E}_{4} \mid W=1\right) \\
& \quad \stackrel{(a)}{\leq} \sum_{\hat{w} \neq 1} \sum_{k} \sum_{\hat{k} \neq k} \operatorname{Pr}\left\{K_{1}=k\right\} \operatorname{Pr}\left\{J_{\hat{w}}=J(\hat{k}) \oplus \bar{P}_{1}\right\}
\end{aligned}
$$

$$
\begin{aligned}
& \quad \times \operatorname{Pr}\left\{\left(U^{N}(\hat{k}), Y^{N}\right) \in \mathcal{T}_{\epsilon}^{N} \mid K_{1}=k, W=1\right\} \\
& \stackrel{(b)}{=} \sum_{\hat{w} \neq 1} \sum_{k} \sum_{\hat{k} \neq k} \sum_{\bar{p}} \sum_{j} \operatorname{Pr}\left\{K_{1}=k\right\} \\
& \quad \times \operatorname{Pr}\left\{J_{\hat{w}}=\bar{p} \oplus j\right\} \operatorname{Pr}\left\{\bar{P}_{1}=\bar{p}, J(\hat{k})=j\right\} \\
& \quad \times \operatorname{Pr}\left\{\left(U^{N}(\hat{k}), Y^{N}\right) \in \mathcal{T}_{\epsilon}^{N} \mid K_{1}=k, W=1\right\} \\
& \stackrel{(\star)}{\leq} 2^{N\left(R_{I}+R_{c}-R_{J}\right)}(1+\hat{\epsilon}) 2^{-N(I(U ; Y)-\delta)} \\
& =(1+\hat{\epsilon}) 2^{N\left(R_{I}-R_{J}+I(U ; X)-I(U ; Y)+2 \delta\right)},
\end{aligned}
$$

where $\hat{\epsilon}>0$ is a fixed number. (a) holds due to the union bound; (b) follows from that $\left(J(\hat{k}), \bar{P}_{1}\right)$ is independent of $J_{\hat{w}}$ given $\hat{w} \neq 1 ;(\star)$ follows from the following analysis using Lemma 1 in [11] or Lemma 1 in [12] (Lemma 1 in [12] is an extension of Lemma 1 in [11]). Note that given $\left(K_{1}=k, \hat{k} \neq k\right), U^{N}(\hat{k})$ is not necessary conditionally independent of $Y^{N}$ or i.i.d. distributed according to $P_{U}$. Let $A$ denote $\left(K_{1}=k, W=1\right)$ for brevity. For sufficiently large $N$, we can obtain that

$$
\begin{aligned}
& \sum_{\hat{k} \neq k} \operatorname{Pr}\left\{\left(U^{N}(\hat{k}), Y^{N}\right) \in \mathcal{T}_{\epsilon}^{N} \mid A\right\} \\
& =\sum_{\hat{k} \neq k} \sum_{\left(\hat{u}^{N}, y^{N}\right) \in \mathcal{T}_{\epsilon}^{N}} \operatorname{Pr}\left\{U^{N}(\hat{k})=\hat{u}^{N}, Y^{N}=y^{N} \mid A\right\} \\
& =\sum_{\hat{k} \neq k} \sum_{\left(\hat{u}^{N}, y^{N}\right) \in \mathcal{T}_{\epsilon}^{N}} \sum_{u^{N}} \\
& \operatorname{Pr}\left\{U^{N}(\hat{k})=\hat{u}^{N}, Y^{N}=y^{N}, U^{N}(k)=u^{N} \mid A\right\} \\
& =\sum_{\hat{k} \neq k} \sum_{\left(\hat{u}^{N}, y^{N}\right) \in \mathcal{T}_{\epsilon}^{N}} \sum_{u^{N}} \\
& \operatorname{Pr}\left\{U^{N}(\hat{k})=\hat{u}^{N} \mid Y^{N}=y^{N}, U^{N}(k)=u^{N}, A\right\} \\
& \times \operatorname{Pr}\left\{U^{N}(k)=u^{N}, Y^{N}=y^{N} \mid A\right\} \\
& \stackrel{(\star)}{\leq} \sum_{\hat{k} \neq k} \sum_{\left(\hat{u}^{N}, y^{N}\right) \in \mathcal{T}_{\epsilon}^{N}} \sum_{u^{N}}(1+\hat{\epsilon}) \prod_{i=1}^{N} P_{U}\left(\hat{u}_{i}\right) \\
& \times \operatorname{Pr}\left\{U^{N}(k)=u^{N}, Y^{N}=y^{N} \mid A\right\} \\
& \leq \sum_{\hat{k} \neq k} \sum_{y^{N}} \sum_{u^{N}}(1+\hat{\epsilon}) 2^{-N(I(U ; Y)-\delta)} \\
& \times \operatorname{Pr}\left\{U^{N}(k)=u^{N}, Y^{N}=y^{N} \mid A\right\} \\
& =\sum_{\hat{k} \neq k}(1+\hat{\epsilon}) 2^{-N(I(U ; Y)-\delta)},
\end{aligned}
$$

where $\hat{\epsilon}>0$ is a fixed number. $(\star)$ follows from Lemma 1 in [12]. Therefore, we can bound $\operatorname{Pr}\left(\mathcal{E}_{4} \mid W=1\right)$ as

$$
\begin{aligned}
& \operatorname{Pr}\left(\mathcal{E}_{4} \mid W=1\right) \\
& \leq \sum_{\hat{w} \neq 1} \sum_{k} \sum_{\hat{k} \neq k} \sum_{\bar{p}} \sum_{j} \operatorname{Pr}\left\{K_{1}=k\right\} \\
& \quad \times \operatorname{Pr}\left\{J_{\hat{w}}=\bar{p} \oplus j\right\} \operatorname{Pr}\left\{\bar{P}_{1}=\bar{p}, J(\hat{k})=j\right\} \\
& \quad \times(1+\hat{\epsilon}) 2^{-N(I(U ; Y)-\delta)} \\
& \leq 2^{N\left(R_{I}+R_{c}-R_{J}\right)}(1+\hat{\epsilon}) 2^{-N(I(U ; Y)-\delta)} .
\end{aligned}
$$

Since $R_{J}>R_{I}+I(U ; X)-I(U ; Y)+2 \delta, \operatorname{Pr}\left(\mathcal{E}_{4} \mid W=\right.$ 1) $\rightarrow 0$ as $N \rightarrow \infty$. As a consequence, due to the union 
bound, $\operatorname{Pr}(\mathcal{E}) \rightarrow 0$ as $N \rightarrow \infty$.

Total Leakage Analysis: The existence of a identification scheme where the mappings $e(\cdot)$ and $d(\cdot)$ are deterministic follows from the existence of a codebook and deterministic mappings from $k_{w}$ to $s_{w}, j\left(k_{w}\right)$. For such mappings, the total leakage can be bounded as

$$
\begin{aligned}
& I\left(S_{W} ; J_{W}\right)+I\left(X^{N}(W) ; J_{W}\right) \\
& \leq 2 I\left(S_{W}, X^{N}(W) ; J\left(K_{W}\right) \oplus \bar{P}_{W}\right) \\
&= 2 H\left(J\left(K_{W}\right) \oplus \bar{P}_{W}\right) \\
& \quad-2 H\left(J\left(K_{W}\right) \oplus \bar{P}_{W} \mid X^{N}(W), S_{W}\right) \\
& \leq 2 \log 2^{N R_{J}}-2 H\left(J\left(K_{W}\right) \oplus \bar{P}_{W} \mid X^{N}(W), S_{W}\right) \\
& \stackrel{(c)}{=} 2 \log 2^{N R_{J}}-2 H\left(\bar{P}_{W} \mid X^{N}(W), S_{W}, J\left(K_{W}\right)\right) \\
& \stackrel{(d)}{=} 0,
\end{aligned}
$$

where $(c)$ follows from our coding scheme that given $X^{n}(W), J\left(K_{W}\right)$ is known; $(d)$ is valid since $\bar{P}_{W}$ is independent of $\left(X^{n}(W), S_{W}, J\left(K_{W}\right)\right)$.

Uniformity of Secret Key Analysis: The uniformity of the secret key can be proved by showing that the codeword index $K_{W}$ for any user $W$ is uniformly distributed. Since $W$ is uniformly distributed in $\left[1: M_{I}\right]$, due to symmetry, it is sufficient to consider $W=1$. For any sequence $u^{N}(k)$ where $k \in\left[1: 2^{N R_{c}}\right]$, there is

$$
\operatorname{Pr}\left\{X^{N}(1) \in \mathcal{T}_{\epsilon}^{N}\left(X \mid u^{N}(k)\right)\right\} \leq 2^{-N(I(U ; X)-\delta)} .
$$

Let $E$ be a random variable such that $E=0$ when there exists a codeword index $k_{1}$ for user $W=1$ satisfying $\left(X^{N}(1), u^{N}\left(k_{1}\right)\right) \in \mathcal{T}_{\epsilon}^{N}$. If no such codeword index exists, then $E=1$. Let $\gamma$ denote $\operatorname{Pr}(E=1)$. According to the error events analysis, we obtain that $\gamma \rightarrow 0$ as $N \rightarrow \infty$.

Similar to $[8,105-106]$, consider the following joint entropy

$$
\begin{aligned}
H\left(K_{1}\right)= & H\left(K_{1}, E\right)-H\left(E \mid K_{1}\right) \geq H\left(K_{1}, E\right)-H(E) \\
\stackrel{(e)}{\geq} & -\sum_{k} \operatorname{Pr}\left(K_{1}=k, E=0\right) \\
& \quad \times \log \left(\operatorname{Pr}\left\{X^{N}(1) \in \mathcal{T}_{\epsilon}^{N}\left(X \mid u^{N}(k)\right)\right\}\right) \\
& -h_{2}(\operatorname{Pr}(E=0)) \\
\geq & N(I(U ; X)-\delta) \sum_{k} \operatorname{Pr}\left(K_{1}=k, E=0\right) \\
& -h_{2}(\operatorname{Pr}(E=0)) \\
= & N(I(U ; X)-\delta)(1-\gamma)-h_{2}(1-\gamma),
\end{aligned}
$$

where we use $h_{2}(\cdot)$ to denote the binary entropy function. (e) follows from that if $K_{1}=k$ and $E=0$ hold, then $X^{N}(1) \in \mathcal{T}_{\epsilon}^{N}\left(X \mid u^{N}(k)\right)$ is valid. Thus $K_{W}$ is close to uniform in the entropy sense. Moreover, the private key is uniformly distributed and independent of the user identity and biometrics. Since the secret key is a combination of the codeword index and part of the private key, this proves that the secret key is close to uniform.

In summary, the following region is achievable

$$
R_{I}+R_{S} \leq R_{P}+I(U ; Y),
$$

$$
R_{I}+I(U ; X) \leq R_{P}+I(U ; Y) .
$$

2) Converse: Let $\boldsymbol{J}$ denote $\left(J_{i}\right)_{i=1}^{M_{I}}$. Define auxiliary random variable $U_{n}=\left(W, S_{W}, P_{W}, J_{W}, X^{n-1}(W)\right)$ for $n \in[1: N]$. Similar to [9, (38)], consider the following joint entropy

$$
\begin{aligned}
& H\left(W, S_{W}\right) \\
& \stackrel{(a)}{=} I\left(W, S_{W} ; P_{W}, \boldsymbol{J}, Y^{N}\right)+H\left(W, S_{W} \mid P_{W}, \boldsymbol{J}, Y^{N}, \hat{W}, \hat{S}\right) \\
& \leq I\left(W, S_{W} ; P_{W}, \boldsymbol{J}, Y^{N}\right)+H\left(W, S_{W} \mid \hat{W}, \hat{S}\right) \\
& \stackrel{(b)}{\leq} I\left(W, S_{W} ; P_{W}, \boldsymbol{J}, Y^{N}\right)+F \\
& \stackrel{(c)}{=} I\left(S_{W} ; \boldsymbol{J} \mid W\right)+I\left(W, S_{W} ; Y^{N}, P_{W} \mid \boldsymbol{J}\right)+F \\
& \stackrel{(d)}{\leq} I\left(S_{W} ; J_{W}, W\right)+I\left(W, S_{W} ; Y^{N}, P_{W} \mid \boldsymbol{J}\right)+F \\
& \stackrel{(e)}{=} I\left(S_{W} ; J_{W}\right)+I\left(W, S_{W} ; Y^{N}, P_{W} \mid \boldsymbol{J}\right)+F \\
& \leq N \delta+H\left(P_{W}\right)+I\left(W, S_{W}, J_{W}, P_{W} ; Y^{N}\right)+F \\
& \stackrel{(f)}{=} N \delta+H\left(P_{W}\right)+I\left(Y^{N} ; W, S_{W}, P_{W}, J_{W}\right)+F \\
& \leq N\left(2 \delta+R_{P}\right)+F \\
& \quad+\sum_{n=1}^{N} I\left(Y_{n} ; W, S_{W}, P_{W}, J_{W}, Y^{n-1}, X^{n-1}(W)\right) \\
& \stackrel{(h)}{=} N\left(2 \delta+R_{P}\right)+F \\
& \quad+\sum_{n=1}^{N} I\left(Y_{n} ; W, S_{W}, P_{W}, J_{W}, X^{n-1}(W)\right) \\
& \quad N\left(2 \delta+R_{P}\right)+\sum_{n=1}^{N} I\left(Y_{n} ; U_{n}\right)+F,
\end{aligned}
$$

where (a) follows from the fact that $(\hat{W}, \hat{S})$ are functions of $\left(P_{W}, \boldsymbol{J}, Y^{N}\right)$; (b) follows from Fano's inequality $H\left(W, S_{W} \mid \hat{W}, \hat{S}\right) \leq F$, where $F=1+\operatorname{Pr}\{(\hat{W}, \hat{S}) \neq$ $\left.\left(W, S_{W}\right)\right\} \log \left(M_{I} M_{S}\right)$; (c) holds since $W$ is independent of $\boldsymbol{J} ;$ (d) is valid since given $W, S_{W}$ is independent of the the helper data of the other users; (e) follows from that $W$ is independent of both $J_{W}$ and $S_{W}$; (f) holds as $Y^{N}$ only correlates to the helper data of the observed user in the database;

(h) holds due to $Y^{n-1}-\left(W, S_{W}, P_{W}, J_{W}, X^{n-1}(W)\right)-Y_{n}$.

Combine the above results, we obtain that

$$
\begin{aligned}
\log & M_{I} M_{S} \\
& \stackrel{(a)}{\leq} \log M_{I}+\min _{w=1,2, \cdots, M_{I}} H\left(S_{w}\right)+N \delta \\
& \leq H(W)+H\left(S_{W} \mid W\right)+N \delta=H\left(W, S_{W}\right)+N \delta \\
& \leq N R_{P}+\sum_{n=1}^{N} I\left(Y_{n} ; U_{n}\right)+F+3 N \delta
\end{aligned}
$$

where (a) follows from the uniformity of the secret key (11c). This gives that

$$
\begin{aligned}
R_{I}+R_{S}-2 \delta & \leq \frac{\log M_{I} M_{S}}{N} \\
& \leq R_{P}+\frac{1}{N} \sum_{n=1}^{N} I\left(Y_{n} ; U_{n}\right)+\frac{F}{N}+3 \delta .
\end{aligned}
$$


For the total leakage, there is

$$
\begin{aligned}
& I\left(S_{W} ; J_{W}\right)+I\left(X^{N}(W) ; J_{W}\right) \\
& \geq I\left(X^{N}(W) ; J_{W}\right)=I\left(X^{N}(W) ; \boldsymbol{J} \mid W\right) \\
& \stackrel{(b)}{=} I\left(X^{N}(W), W ; \boldsymbol{J}\right) \\
& \geq H\left(X^{N}(W), W\right)-H\left(X^{N}(W), S_{W}, W \mid \boldsymbol{J}\right) \\
& =H(W)+H\left(X^{N}(W)\right)-H\left(S_{W}, W \mid \boldsymbol{J}, Y^{N}, P_{W}\right) \\
& -I\left(Y^{N}, P_{W} ; S_{W}, W \mid \boldsymbol{J}\right)-H\left(X^{N}(W) \mid S_{W}, W, \boldsymbol{J}\right) \\
& \text { (c) } \\
& \stackrel{(c)}{\geq} H(W)+I\left(X^{N}(W) ; S_{W}, W, \boldsymbol{J}\right)-H\left(S_{W}, W \mid \hat{S}, \hat{W}\right) \\
& -I\left(Y^{N}, P_{W} ; S_{W}, W, \boldsymbol{J}\right) \\
& \stackrel{(d)}{\geq} H(W)+I\left(X^{N}(W) ; S_{W}, W, J_{W}\right)-F \\
& -I\left(Y^{N}, P_{W} ; S_{W}, W, J_{W}\right) \\
& \stackrel{(e)}{\geq} N\left(R_{I}-\delta\right)+I\left(X^{N}(W) ; S_{W}, W, J_{W} \mid Y^{N}\right)-F \\
& -I\left(P_{W} ; S_{W}, W, J_{W} \mid Y^{N}\right) \\
& =N\left(R_{I}-\delta\right)+I\left(X^{N}(W) ; S_{W}, W, J_{W} \mid Y^{N}\right)-F \\
& -H\left(P_{W} \mid Y^{N}\right)+H\left(P_{W} \mid S_{W}, W, J_{W}, Y^{N}\right) \\
& \geq N\left(R_{I}-R_{P}-2 \delta\right) \\
& +I\left(X^{N}(W) ; S_{W}, W, J_{W}, P_{W} \mid Y^{N}\right)-F \\
& =N\left(R_{I}-R_{P}-2 \delta\right)-I\left(Y^{N} ; S_{W}, W, J_{W}, P_{W}\right) \\
& +I\left(X^{N}(W), Y^{N} ; S_{W}, W, J_{W}, P_{W}\right)-F \\
& \stackrel{(f)}{=} N\left(R_{I}-R_{P}-2 \delta\right)+I\left(X^{N}(W) ; S_{W}, W, J_{W}, P_{W}\right) \\
& -I\left(Y^{N} ; S_{W}, W, J_{W}, P_{W}\right)-F \\
& =N\left(R_{I}-R_{P}-2 \delta\right) \\
& +\sum_{n=1}^{N} I\left(X_{n}(W) ; S_{W}, W, P_{W}, J_{W}, X^{n-1}(W)\right) \\
& -\sum_{n=1}^{N} I\left(Y_{n} ; S_{W}, W, P_{W}, J_{W}, Y^{n-1}\right)-F \\
& \stackrel{(g)}{\geq} N\left(R_{I}-R_{P}-2 \delta\right) \\
& +\sum_{n=1}^{N} I\left(X_{n}(W) ; U_{n}\right)-\sum_{n=1}^{N} I\left(Y_{n} ; U_{n}\right)-F,
\end{aligned}
$$

where (b) follows from that $W$ is independent of $\boldsymbol{J}$; (c) follows from the fact that $(\hat{W}, \hat{S})$ are functions of $\left(P_{W}, \boldsymbol{J}, Y^{N}\right)$; (d) follows from that the $X^{N}(W)$ and $Y^{N}$ only correlate to the helper data $J_{W}$ of user $W$ in the database, and Fano's inequality; (e) and (f) hold due to the Markov chain $Y^{N}-X^{N}(W)-\left(S_{W}, W, J_{W}, P_{W}\right)$; (g) is valid since $Y^{n-1}-\left(S_{W}, W, J_{W}, P_{W}, X^{n-1}(W)\right)-Y_{n}$.

Combine the above result with (11e), we can obtain that

$$
\begin{aligned}
R_{I}+ & \frac{1}{N} \sum_{n=1}^{N} I\left(X_{n}(W) ; U_{n}\right) \\
& \leq R_{P}+\frac{1}{N} \sum_{n=1}^{N} I\left(Y_{n} ; U_{n}\right)-\frac{1}{N} F+3 \delta .
\end{aligned}
$$

Let $Q$ be a uniform random variable on $[1: N]$ and independent of everything else. Define $U=\left(U_{Q}, Q\right)$ and
$P_{U X_{Q} Y_{Q}}=Q_{Y_{Q} \mid X_{Q}} P_{U \mid X_{Q}}$. Note that $U-X_{Q}(W)-Y_{Q}$ still holds. As $\left(X_{Q}, Y_{Q}\right)$ has the same joint distribution as $(X, Y)$, let $\delta \rightarrow 0$, we can obtain that $\left(R_{I}, R_{S}, R_{P}\right) \in \mathcal{R}^{S}$ from (26) and (28) by the cardinality bounding arguments. This completes the proof of the backward direction.

\section{Proof of Theorem 2}

The proof for Theorem 2 is similar to that of Theorem 1 . Here we provide the details for completeness.

1) Achievability: Fix the conditional probability $P_{U V \mid X}$. Thus there is $P_{U V X Y}=Q_{X} Q_{Y \mid X} P_{U V \mid X}$. Let the number of enrolled users be $M_{I}=2^{N R_{I}}$. Additionally, fix a $\delta>0$.

Codebook generation: Pick a rate tuple $\left(R_{I}, R_{S}, R_{P}\right) \in$ $\mathcal{R}^{T}$ such that $R_{P} \geq R_{I}+I(U, V ; X)-I(U, V ; Y)+5 \delta$ and $R_{S}=R_{P}-R_{I}+I(U, V ; Y)-3 \delta$. Generate $2^{N R_{C}}$ i.i.d. codewords $u^{N}\left(k_{1}\right)$ according to $P_{U}$, where we fix $R_{C}=$ $I(U ; X)+\delta$. For each $k_{1} \in\left[1: 2^{N R_{C}}\right]$, generate $2^{N R_{V}}$ codewords $v^{N}\left(k_{1}, k_{2}\right)$ according to $\prod_{i=1}^{N} P_{V \mid U=u_{i}\left(k_{1}\right)}$, where we fix $R_{V}=I(V ; X \mid U)+\delta$. Then, for each pair $\left(k_{1}, k_{2}\right)$, generate a secret label $s\left(k_{1}, k_{2}\right)=\left(k_{1}, k_{2}\right)$ and a helper data label $j\left(k_{1}, k_{2}\right)$ which is uniformly generated from $\left[1: 2^{N R_{J}}\right]$ at random. Additionally, we fix $R_{J}=R_{I}+I(U, V ; X)-$ $I(U, V ; Y)+5 \delta$. Note that $R_{J}$ does not necessarily equal $R_{C}+R_{V}$.

Enrollment: For each user $w \in\left[1: 2^{N R_{I}}\right]$, the encoder firstly looks for a cluster centroid $u^{N}\left(k_{1 w}\right)$ such that $\left(u^{N}\left(k_{1 w}\right), x^{N}(w)\right) \in \mathcal{T}_{\epsilon}^{N}$, and put the user index $w$ in $\mathcal{B}\left(c_{w}\right)$ where $c_{w}=k_{1 w}$. If no such $c_{w}$ exists, randomly choose a cluster index from $\left[1: 2^{N R_{C}}\right]$. If there is more than one such $c_{w}$, randomly choose one from them. Then the encoder looks for a codeword $v^{N}\left(k_{1 w}, k_{2 w}\right)$ such that $\left(u^{N}\left(k_{1 w}\right), v^{N}\left(k_{1 w}, k_{2 w}\right), x^{N}(w)\right) \in \mathcal{T}_{\epsilon}^{N}$. If no such $k_{2 w}$ exists, randomly choose one from $\left[1: 2^{N R_{V}}\right]$. If there is more than one such $k_{2 w}$, randomly choose one from them. Given the private key of this user $p_{w}$, we divide it into two parts, $\bar{p}_{w}$ and $\tilde{p}_{w}$, such that $p_{w}=\left(\bar{p}_{w}, \tilde{p}_{w}\right), \bar{p}_{w} \in\left[1: 2^{N R_{J}}\right]$ and $\tilde{p}_{w} \in$ $\left[1: 2^{N\left(R_{P}-R_{J}\right)}\right]$. With $\left(k_{1 w}, k_{2 w}\right)$, the encoder produces the corresponding helper data label $j\left(k_{1 w}, k_{2 w}\right)$, which is masked by $\bar{p}_{w}$ so that we obtain $j_{w}=j\left(k_{1 w}, k_{2 w}\right) \oplus \bar{p}_{w}$. The helper data index $j_{w}$ is then stored in $k_{1 w}$-th cluster at the location of $w$. A secret key $s_{w}$ is also generated. Let the secret key be generated as $s_{w}=\left(s\left(k_{1 w}, k_{2 w}\right), \tilde{p}_{w}\right)$.

Identification: After receiving the observation $y^{N}$ and having access to the private key $p$ of the observed user $w$, the decoder firstly looks for a list of possible cluster centroid indices $\mathcal{L}\left(y^{N}\right)$ such that for each $c_{l} \in \mathcal{L}\left(y^{N}\right)$, $\left(u^{N}\left(c_{l}\right), y^{N}\right) \in \mathcal{T}_{\epsilon}^{N}$. Next, the decoder checks the members in the clusters and looks for a unique triple $\left(\hat{w}, \hat{k}_{1}, \hat{k}_{2}\right)$ such that $\hat{k}_{1} \in \mathcal{L}, \hat{w} \in \mathcal{B}\left(\hat{k}_{1}\right), j_{\hat{w}}=j\left(\hat{k}_{1}, \hat{k}_{2}\right) \oplus \bar{p}$ and $\left(u^{N}\left(\hat{k}_{1}\right), v^{N}\left(\hat{k}_{1}, \hat{k}_{2}\right), y^{N}\right) \sim \mathcal{T}_{\epsilon}^{N}$. The estimated secret key is $\hat{s}=\left(s\left(\hat{k}_{1}, \hat{k}_{2}\right), \tilde{p}\right)$.

Analysis: Assume that the user $W$ is observed. Let $P_{W}$ be the private key, $\left(K_{1 W}, K_{2 W}\right)$ be the codeword index pair determined by the encoder, $J\left(K_{1 W}, K_{2 W}\right)$ be the corresponding helper data label, $J_{W}$ and $S_{W}$ be the actual masked helper data and secret key respectively. Consider the 
following error events:

$$
\begin{aligned}
\mathcal{E}_{11}=\{ & \left.\left(U^{N}\left(k_{1}\right), X^{N}(W)\right) \notin \mathcal{T}_{\epsilon}^{N}, \forall k_{1} \in\left[1: 2^{N R_{C}}\right]\right\}, \\
\mathcal{E}_{12}=\{ & \left(U^{N}\left(K_{1 W}\right), V^{N}\left(K_{1 W}, k_{2}\right), X^{N}(W)\right) \notin \mathcal{T}_{\epsilon}^{N}, \\
& \left.\forall k_{2} \in\left[1: 2^{N R_{V}}\right]\right\}, \\
\mathcal{E}_{13}=\{ & \left.\left(U^{N}\left(K_{1 W}\right), V^{N}\left(K_{1 W}, K_{2 W}\right), Y^{N}\right) \notin \mathcal{T}_{\epsilon}^{N}\right\}, \\
\mathcal{E}_{21}=\{ & \exists \neq W, J_{\hat{w}}=J\left(K_{1 W}, K_{2 W}\right) \oplus \bar{P}_{W}, \\
& \left.\left(U^{N}\left(K_{1 W}\right), V^{N}\left(K_{1 W}, K_{2 W}\right), Y^{N}\right) \in \mathcal{T}_{\epsilon}^{N}\right\} . \\
\mathcal{E}_{22}=\{ & \left(\hat{k}_{2}, \hat{w}\right), \hat{k}_{2} \neq K_{2 W}, \hat{w} \neq W \\
& J_{\hat{w}}=J\left(K_{1 W}, \hat{k}_{2}\right) \oplus \bar{P}_{W}, \\
& \left.\left(U^{N}\left(K_{1 W}\right), V^{N}\left(K_{1 W}, \hat{k}_{2}\right), Y^{N}\right) \in \mathcal{T}_{\epsilon}^{N}\right\}, \\
\mathcal{E}_{23}=\{ & \left(\hat{k}_{1}, \hat{w}\right), \hat{k}_{1} \in \mathcal{L}\left(Y^{N}\right), \hat{k}_{1} \neq K_{1 W}, \hat{w} \neq W, \\
& \left(U^{N}\left(\hat{k}_{1}\right), V^{N}\left(\hat{k}_{1}, \hat{k}_{2}\right), Y^{N}\right) \in \mathcal{T}_{\epsilon}^{N}, \\
& \left.J_{\hat{w}}=J\left(\hat{k}_{1}, \hat{k}_{2}\right) \oplus \bar{P}_{W} \text { for some } \hat{k}_{2}\right\} .
\end{aligned}
$$

If none of the above events occur, the identification will be successful, thus we have the following identification error event

$$
\mathcal{E}=\mathcal{E}_{11} \cup \mathcal{E}_{12} \cup \mathcal{E}_{13} \cup \mathcal{E}_{21} \cup \mathcal{E}_{22} \cup \mathcal{E}_{23} .
$$

Since $R_{C}>I(U ; X), \operatorname{Pr}\left(\mathcal{E}_{11}\right) \rightarrow 0$ as $N \rightarrow \infty$ due to the covering lemma [10, Lemma 3.3]. As $R_{V}>I(V ; X \mid U)$, $\operatorname{Pr}\left(\mathcal{E}_{11}^{c} \cap \mathcal{E}_{12}\right) \rightarrow 0$ as $N \rightarrow \infty$ according the covering lemma [10, Lemma 3.3]. $\operatorname{Pr}\left(\mathcal{E}_{11}^{c} \cap \mathcal{E}_{12}^{c} \cap \mathcal{E}_{13}\right) \rightarrow 0$ as $N \rightarrow \infty$ according to Markov lemma [10, p.27].

In the following error events analysis, due to the symmetry and without losing generality, we consider the case when the user identity is $W=1$. We use $\left(k_{11}, k_{21}\right)$ to denote the codeword index pair for user $W=1$.

Consider the probability of error event $\mathcal{E}_{21}$, we can obtain that

$$
\begin{aligned}
\operatorname{Pr}\left(\mathcal{E}_{21} \mid W=1\right) \leq & \sum_{\hat{w} \neq 1} \sum_{\bar{p}} \sum_{j} \operatorname{Pr}\left\{J_{\hat{w}}=\bar{p} \oplus j\right\} \\
& \times \operatorname{Pr}\left\{\bar{P}_{1}=\bar{p}, J\left(k_{11}, k_{21}\right)=j\right\} \\
\leq & 2^{N\left(R_{I}-R_{J}\right)} .
\end{aligned}
$$

Since $R_{J}>R_{I}, \operatorname{Pr}\left(\mathcal{E}_{21} \mid W=1\right) \rightarrow 0$ as $N \rightarrow \infty$.

The probability $\operatorname{Pr}\left(\mathcal{E}_{22} \mid W=1\right)$ can be bounded as

$$
\begin{gathered}
\operatorname{Pr}\left(\mathcal{E}_{22} \mid W=1\right) \\
\leq \sum_{k_{11}} \sum_{k_{21}} \operatorname{Pr}\left\{K_{11}=k_{11}, K_{21}=k_{21}\right\} \\
\quad \sum_{\hat{w} \neq 1} \sum_{\hat{k}_{2} \neq k_{21}} \operatorname{Pr}\left\{J_{\hat{w}}=J\left(k_{11}, \hat{k}_{2}\right) \oplus \bar{P}_{1}\right\} \\
\quad \times \operatorname{Pr}\left\{\left(U^{N}\left(k_{11}\right), V^{N}\left(k_{11}, \hat{k}_{2}\right), Y^{N}\right) \in \mathcal{T}_{\epsilon}^{N}\right. \\
\left.\quad \mid K_{11}=k_{11}, K_{21}=k_{21}, W=1\right\} \\
\stackrel{(\star)}{\leq} 2^{N\left(R_{I}-R_{J}+R_{V}\right)}(1+\hat{\epsilon}) 2^{-N(I(V ; Y \mid U)-\delta)} \\
=(1+\hat{\epsilon}) 2^{N\left(R_{I}-R_{J}+I(V ; X \mid U)-I(V ; Y \mid U)+2 \delta\right)},
\end{gathered}
$$

where $\hat{\epsilon}>0$ is a fixed number. ( $\star$ ) follows from Lemma 1 .

As for the probability of the last error event, for sufficiently large enough $N$, we have that

$$
\operatorname{Pr}\left(\mathcal{E}_{23} \mid W=1\right)
$$

$$
\begin{aligned}
& \leq \sum_{\hat{w} \neq 1} \sum_{k_{11}} \operatorname{Pr}\left\{K_{11}=k_{11}\right\} \\
& \times \operatorname{Pr}\left\{\exists \hat{k}_{1} \neq k_{11}, \hat{k}_{1} \in \mathcal{L}\left(Y^{N}\right),\right. \\
& J_{\hat{w}}=J\left(\hat{k}_{1}, \hat{k}_{2}\right) \oplus \bar{P}_{1}, \\
& \left(U^{N}\left(\hat{k}_{1}\right), V^{N}\left(\hat{k}_{1}, \hat{k}_{2}\right), Y^{N}\right) \in \mathcal{T}_{\epsilon}^{N}, \\
& \text { for some } \left.\hat{k}_{2} \mid K_{11}=k_{11}, W=1\right\} \\
& \leq \sum_{\hat{w} \neq 1} \sum_{k_{11}} \sum_{\hat{k}_{1} \neq k_{11}} \sum_{\hat{k}_{2}} \operatorname{Pr}\left\{K_{11}=k_{11}\right\} \\
& \times \operatorname{Pr}\left\{J_{\hat{w}}=J\left(\hat{k}_{1}, \hat{k}_{2}\right) \oplus \bar{P}_{1}\right\} \\
& \times \operatorname{Pr}\left\{\left(U^{N}\left(\hat{k}_{1}\right), V^{N}\left(\hat{k}_{1}, \hat{k}_{2}\right), Y^{N}\right) \in \mathcal{T}_{\epsilon}^{N}\right. \\
& \left.\mid K_{11}=k_{11}, W=1\right\} \\
& =\sum_{\hat{w} \neq 1} \sum_{k_{11}} \sum_{\hat{k}_{1} \neq k_{11}} \sum_{\hat{k}_{2}} \operatorname{Pr}\left\{K_{11}=k_{11}\right\} \\
& \times \operatorname{Pr}\left\{J_{\hat{w}}=J\left(\hat{k}_{1}, \hat{k}_{2}\right) \oplus \bar{P}_{1}\right\} \\
& \times \operatorname{Pr}\left\{\left(U^{N}\left(\hat{k}_{1}\right), Y^{N}\right) \in \mathcal{T}_{\epsilon}^{N} \mid K_{11}=k_{11}, W=1\right\} \\
& \times \operatorname{Pr}\left\{V^{N}\left(\hat{k}_{1}, \hat{k}_{2}\right) \in \mathcal{T}_{\epsilon}^{N}\left(V \mid U^{N}\left(\hat{k}_{1}\right), Y^{N}\right)\right. \\
& \left.\mid K_{11}=k_{11}, W=1\right\} \\
& \stackrel{(\star \star)}{\leq} \sum_{\hat{w} \neq 1} \sum_{k_{11}} \sum_{\hat{k}_{1} \neq k_{11}} \sum_{\hat{k}_{2}} \operatorname{Pr}\left\{K_{11}=k_{11}\right\} \\
& \times \operatorname{Pr}\left\{J_{\hat{w}}=J\left(\hat{k}_{1}, \hat{k}_{2}\right) \oplus \bar{P}_{1}\right\} \\
& \times(1+\hat{\epsilon}) 2^{-N(I(U ; Y)-\delta)} 2^{-N(I(V ; Y \mid U)-\delta)} \\
& \leq 2^{N\left(R_{I}+R_{C}+R_{V}-R_{J}\right)}(1+\hat{\epsilon}) 2^{-N(I(U, V ; Y)-2 \delta)} \\
& =(1+\hat{\epsilon}) 2^{N\left(R_{I}-R_{J}+I(U, V ; X)-I(U, V ; Y)+4 \delta\right)},
\end{aligned}
$$

where $\hat{\epsilon}>0$ is a fixed number. ( $\star \star)$ follows from similar analysis of bounding $\operatorname{Pr}\left(\mathcal{E}_{4} \mid W=1\right)$ and the fact that given $\left(K_{11}=k_{11}, W=1, \hat{k}_{1} \neq k_{11}, U^{N}\left(\hat{k}_{1}\right)=u^{N}\left(\hat{k}_{1}\right)\right)$, $V^{N}\left(\hat{k}_{1}, \hat{k}_{2}\right)$ is independent of $Y^{N}$ and i.i.d. distributed according to $\prod_{i=1}^{N} P_{V \mid U}\left(\cdot \mid u_{i}\left(\hat{k}_{1}\right)\right)$. Since $R_{J}>R_{I}+$ $I(U, V ; X)-I(U, V ; Y)+4 \delta, \operatorname{Pr}\left(\mathcal{E}_{23} \mid W=1\right) \rightarrow 0$ as $N \rightarrow \infty$. Consequently, due to the union bound, $\operatorname{Pr}(\mathcal{E}) \rightarrow 0$ as $N \rightarrow \infty$.

Similar to (20), the total leakage can be bounded as $I\left(S_{W} ; J_{W}\right)+I\left(X^{N}(W) ; J_{W}\right) \leq 0$.

The uniformity of the secret key can be proved by showing that the codeword index pair $\left(K_{1 W}, K_{2 W}\right)$ for any user $W$ is uniformly distributed. Since $W$ is uniformly distributed in $\left[1: M_{I}\right]$, due to symmetry, it is sufficient to consider $W=1$. For any codewords $u^{N}$ and $v^{N}$, there is

$$
\operatorname{Pr}\left\{\left(X^{N}(1), u^{N}, v^{N}\right) \in \mathcal{T}_{\epsilon}^{N}\right\} \leq 2^{-N(I(U, V ; X)-\delta)} .
$$

Let $E_{2}=0$ be a random variable such that when there exists a codeword index pair $\left(k_{11}, k_{21}\right)$ for user $W=1$ satisfying $\left(x^{N}(1), u^{N}\left(k_{11}\right), v^{N}\left(k_{11}, k_{12}\right)\right) \in \mathcal{T}_{\epsilon}^{N}$. If there is no such codeword index pair, then $E_{2}=1$. Let $\gamma_{2}$ denote $\operatorname{Pr}\left(E_{2}=\right.$ $1)$. Then according to the error events analysis, we can obtain that $\gamma_{2} \rightarrow 0$ as $N \rightarrow \infty$. Now we consider the following joint entropy

$$
\begin{aligned}
& H\left(K_{11}, K_{21}\right) \\
& =H\left(K_{11}, K_{21}, E_{2}\right)-H\left(E_{2} \mid K_{11}, K_{21}\right)
\end{aligned}
$$




$$
\begin{aligned}
& \geq H\left(K_{11}, K_{21}, E_{2}\right)-H\left(E_{2}\right) \\
& \begin{array}{l}
\stackrel{(a)}{\geq}-\sum_{k_{1}} \sum_{k_{2}} \operatorname{Pr}\left(K_{11}=k_{1}, K_{21}=k_{2}, E_{2}=0\right) \\
\quad \times \log \left\{\operatorname{Pr}\left(x^{N}(1) \in \mathcal{T}_{\epsilon}^{N}\left(X \mid u^{N}\left(k_{1}\right), v^{N}\left(k_{1}, k_{2}\right)\right)\right\}\right.
\end{array} \\
& \quad-h_{2}\left(\operatorname{Pr}\left(E_{2}=0\right)\right) \\
& \geq \sum_{k_{1}} \sum_{k_{2}} \operatorname{Pr}\left(K_{11}=k_{1}, K_{21}=k_{2}, E_{2}=0\right) \\
& \quad \times N(I(U, V ; X)-\delta)-h_{2}(\operatorname{Pr}(E=0))
\end{aligned}
$$

where (a) follows from that if $\left(K_{11}=k_{1}, K_{21}=k_{2}, E_{2}=\right.$ $0)$ holds, then $X^{N}(1) \in \mathcal{T}_{\epsilon}^{N}\left(X \mid u^{N}\left(k_{1}\right), v^{N}\left(k_{1}, k_{2}\right)\right)$ is valid. Combine this result with $R_{C}+R_{V}=I(U, V ; X)+2 \delta$, we can conclude that $\left(K_{1 W}, K_{2 W}\right)$ is close to uniformly distributed. Use similar argument in the proof of the singlestage setting, we prove that the secret key is close to uniform.

In summary, the following region is achievable

$$
\begin{aligned}
R_{I}+R_{S} & \leq R_{P}+I(U, V ; Y), \\
R_{I}+I(U, V ; X) & \leq R_{P}+I(U, V ; Y) .
\end{aligned}
$$

2) Converse: Define auxilary random variables $U_{n}=$ $\left(W, X^{n-1}(W)\right)$ and $V_{n}=\left(S_{W}, J_{W}, P_{W}\right)$ for $n \in[1$ : $N]$. Let $\boldsymbol{J}$ denote $\left(J_{i}\right)_{i=1}^{M_{I}}$. Additionally, define $F=1+$ $\operatorname{Pr}\left\{(\hat{W}, \hat{S}) \neq\left(W, S_{W}\right)\right\} \log \left(M_{I} M_{S}\right)$.

Similar to (24), consider the following joint entropy

$$
\begin{aligned}
H\left(W, S_{W}\right)= & N\left(2 \delta+R_{P}\right)+F \\
& +\sum_{n=1}^{N} I\left(Y_{n} ; W, S_{W}, P_{W}, J_{W}, X^{n-1}(W)\right) \\
= & N\left(2 \delta+R_{P}\right)+\sum_{n=1}^{N} I\left(Y_{n} ; U_{n}, V_{n}\right)+F .
\end{aligned}
$$

Assume the tuple $\left(R_{I}, R_{S}, R_{P}\right)$ is achievable, then $F<$ $1+\delta \log \left(M_{I} M_{S}\right)$. Similar to (25), we have that

$$
\begin{aligned}
\log M_{I} M_{S} & \leq H\left(W, S_{W}\right)+N \delta \\
& \leq N R_{P}+\sum_{n=1}^{N} I\left(Y_{n} ; U_{n}, V_{n}\right)+F+3 N \delta .
\end{aligned}
$$

Similar to the proof of the single-stage setting, we can use the cardinality bounding argument to complete the proof for the backward direction.

\section{Example}

Now we consider an example of the single-stage setting to illustrate the tradeoff between the identification rate and secret key rate. Assume a binary symmetric source, i.e., Bernoulli distributed with probability $\frac{1}{2}$. Let the observation channel be a binary symmetric channel with crossover probability $q$. Moreover, assume $H(X \mid U)=h_{2}(p)$ for some $p \in\left(0, \frac{1}{2}\right)$, then from Mrs. Gerber's Lemma [13], we know that $H(Y \mid U) \geq h_{2}(p * q)$, where $p * q=p(1-q)+q(1-p)$.

To illustrate the properties of the achievable region, we fix the private key rate as $R_{P}=r$. We plot the resulting

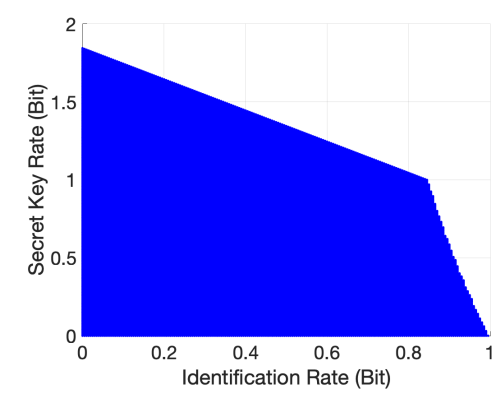

Fig. 3. Rate projections, $q=0.1$ and $r=1$.

achievable rate pair $\left(R_{I}, R_{S}\right)$ for $q=0.1$. In this case, the achievable region becomes

$$
\begin{aligned}
\left.\mathcal{R}^{S}\right|_{R_{P}=r}=\left\{\left(R_{I}, R_{S}\right):\right. & R_{I}+R_{S} \leq r+1-h_{2}(p * q), \\
& \left.R_{I}-h_{2}(p)\right) \leq r-h_{2}(p * q), \\
& \text { for some } P_{U X Y}=Q_{X} Q_{Y \mid X} P_{U \mid X}, \\
& \text { and }|\mathcal{U}| \leq|\mathcal{X}|+1\} .
\end{aligned}
$$

Observe Fig. 3, we can see that there is a tradeoff relationship between the identification rate and the secret rate.

\section{ACKNOWLEDGEMENT}

The work was supported in part by the Swedish Research Council (VR) under grant 2016-03853 and the SRA ICT TNG through the Swedish Government.

\section{REFERENCES}

[1] B. Schneier, "Inside risk: The uses and abuses of biometrics," Comm. ACM, vol. 42, no. 8, pp. 136-1999, 1999.

[2] F. Willems, T. Kalker, J. Goseling, and J. P. Linnartz, "On the capacity of a biometrical identification system," in Proc. IEEE Int. Symp. Inf. Theory, Jun. 2003, pp. 82-87.

[3] E. Tuncel, "Capacity/storage tradeoff in high-dimensional identification systems," in Proc. IEEE Int. Symp. Inf. Theory, Jul. 2006, pp. 1929-1933.

[4] F. Farhadzadeh and F. M. J. Willems, "Identification rate, search and memory complexity tradeoff: Fundamental limits," IEEE Trans. Inf. Theory, vol. 62, no. 11, pp. 6173-6188, Nov. 2016.

[5] M. T. Vu, T. J. Oechtering, and M. Skoglund, "Hierarchical identification with pre-processing," in Proc. IEEE Int. Symp. Inf. Theory, Jun. 2017, pp. 2746-2750.

[6] M. T. Vu, "Perspectives on identification systems," Ph.D. dissertation, KTH, Information Science and Engineering, 2019, qC 20190708.

[7] N. K. Ratha, S. Chikkerur, J. H. Connell, and R. M. Bolle, "Generating cancelable fingerprint templates," IEEE Trans. Pattern Anal. Mach. Intell., vol. 29, no. 4, pp. 561-572, 2007.

[8] T. Ignatenko and F. M. J. Willems, "Biometric systems: Privacy and secrecy aspects," IEEE Tran. Inf. Forensics Security, vol. 4, no. 4, pp. 956-973, Dec. 2009.

[9] — , "Fundamental limits for privacy-preserving biometric identification systems that support authentication," IEEE Trans. Inf. Theory, vol. 61, no. 10, pp. 5583-5594, Oct 2015.

[10] A. E. Gamal and Y. H. Kim, Network information theory. Cambridge university press, 2011.

[11] P. Minero, S. H. Lim, and Y.-H. Kim, "A unified approach to hybrid coding," IEEE Trans. Inf. Theory, vol. 61, no. 4, pp. 1509-1523, 2015.

[12] M. T. Vu, T. J. Oechtering, M. Wiese, and M. Skoglund, "Cooperative successive refinement with side information," manuscript in preparation. [Online]. Available: https://people.kth.se/ mtvu/jnlp. pdf

[13] A. Wyner, "A theorem on the entropy of certain binary sequences and applications-i," IEEE Trans. Inf. Theory, vol. 19, no. 6, pp. 769-772, 1973. 